\title{
The evolution of robotic thoracic surgery
}

\author{
Carmelina C. Zirafa, Gaetano Romano, Teresa Hung Key, Federico Davini, Franca Melfi \\ Minimally Invasive and Robotic Thoracic Surgery, Robotic Multispecialty Center of Surgery, University Hospital of Pisa, Pisa, Italy \\ Correspondence to: Carmelina C. Zirafa. Minimally Invasive and Robotic Thoracic Surgery, Robotic Multispecialty Center of Surgery, University \\ Hospital of Pisa, Via Paradisa 2, 56124 Pisa, Italy. Email: c.zirafa@gmail.com.
}

\begin{abstract}
Robotic surgery has the features to represent the future of surgery, considering the rapid evolution of its technology and the resulting in the surgical field. In the last years, the robotic technique in thoracic surgery has progressively become widespread in the word, particularly for the treatment of the mediastinal and pulmonary lesions. The development of technology in the robotic system has been associated with the improvement of intraoperative and postoperative results. Due to the satisfying results and increasing experience and confidence with the robotic technique, surgeons are consequently enlarging the surgical indication, moving to increasingly challenging cases. Thoracic robotic surgery is being affirmed as a safe technique also for those complex cases, which in the past were considered a matter solely for open surgery. In fact, robotic surgery is increasingly associated with positive surgical results and guarantees less traumatism and a fast recovery to the patients. These positive results have resulted from the evolution of the technique, which has developed in parallel with the evolution of the technology, exploiting to its best the latest features of the robotic system. These features, such as the fluorescence-detection tool or the robotic stapler, have been aiding the surgeon to maximise the safety and feasibility of the application of the robotic technique to thoracic surgery.
\end{abstract}

Keywords: Robotic lobectomy; robotic thymectomy; surgical technique; technology

Submitted Feb 25, 2019. Accepted for publication Mar 04, 2019.

doi: 10.21037 /acs.2019.03.03

View this article at: http://dx.doi.org/10.21037/acs.2019.03.03

\section{Introduction}

Robotic surgery is considered by various authors the future of surgery, due to its impact with radical changes obtained in the surgical field in the last years.

After about sixty years from the coining in 1920 of the term "robot", which means forced labour by the Czech author Capek, the first robot-assisted surgical procedure was reported (1). In the beginning, the robotic systems were exclusively active systems, characterized by an autonomous work under pre-operative planned tasks. Examples of active robotic systems are ROBODOC (Integrated Surgical Systems, Sacramento, CA, USA), used for orthopaedic procedures, and PROBOT, used for urological surgery (2).

Later in the 1990s, more complex platforms which went under the name of master-slave systems, were developed. These platforms were characterized by the loss of the preprogrammed and autonomous function, thus becoming completely depending on the surgeon's actions. Initially, this technology was created under NASA's idea to ensure surgical assistance to astronauts in space, thanks to the telepresence, avoiding the physical presence of a surgeon. Consequently, NASA's Ames Research Centre, which was then joined by Stanford in 1990s, designed the first phase robotic prototype based on telesurgery, which served as the starting platform for the future systems. The real turning point for the surgical robotic field was determined a few years later with the commercialisation of the first FDA approved robotic platforms for civilian use, AESOP (Computer Motion, Inc., Goleta, CA, USA), which combined the tele-manipulator with a foot pedal, later replaced by Zeus. The Zeus system was initially designed for cardiac surgery even though its use for other specialties was recognized as feasible; at a similar time, the da Vinci system (Intuitive Surgical, Sunnyvale, CA, USA) was also introduced into the market. In 2003 with the merger 
between Computer Motion and Intuitive Surgical, the da Vinci became the only robotic surgical platform in use on the market $(3,4)$.

\section{Evolution of the robotic system}

The da Vinci system, constituted by three main components (the vision cart, the master console, from which the surgeon controls robotic instruments and the surgical cart from which the robotic arms extend completed by the robotic surgical instruments), in the course of the years has evolved into various models characterized by tri-dimensional high definition vision, with magnification of image up to 10 times, 7 degrees of freedom of the instruments and filtration of physiologic hand tremor (5). Following the technologic improvement, the latest system, da Vinci Xi permits a faster docking, with guided targeting and a rapid undocking, resulting in an overall reduction of the operative time. Moreover, thanks to the $\mathrm{Xi}$ innovative overhead architecture and the lowered diameter of the trocar $(8 v s .12 \mathrm{~mm})$, during surgical procedure there is a reduction of arm collisions with consequent less traumatism for the patient.

These and several other new technological elements introduced in the robotic system over the years have allowed a progressive enlargement of the application of robotic surgery in the thoracic field.

The innovative features of the robotic system were further implemented by the elaboration of a fluorescencedetection tool, optionally introduced in Si System in 2009, with the name of "Firefly", and later incorporated as a standard tool in Xi system. After the injection of indocyanine green (ICG), applying the intraoperative near-infrared fluorescence (INIF) imaging system present in the robotic system, the surgeon is able to clearly identify the vessels, the intersegmental line during segmentectomy, to localize the thoracic duct in a chylothorax or to detect neoplasms. This tool can be considered as a way of overstepping the lack of tactile feedback of the surgical field (6).

An additional recent technical innovation, only available in $\mathrm{Xi}$ robotic system, has been the robotic staplers introduced in 2014. Vascular resection during major lung resection represents a crucial step in the thoracic surgical procedure, being potentially correlated with intra-operative disasters. Thanks to the introduction of the robotic stapler, if difficulties in positioning the endoscopic stapler by the assistant occur, the surgeon can directly perform vascular resection from the console in complete autonomy (7).

The introduction and the running evolution of robotic technologies in thoracic surgery has allowed the widespread employment of the application of minimally invasive surgery and the expansion of the indications in several benign and neoplastic conditions.

The new computer-assisted technology associated with a properly qualified surgical team can be an instrument that facilitates the performance of high-quality surgery while respecting the core principles of surgery. The training of the surgeons, the anesthesiologists and the nurses become an essential first step in order to achieve fulfilling surgical results, containing the cost of the procedures.

The introduction of the simulator has represented an important role in the training program, a step which is fundamental for surgeons to acquire and refine the skills needed to perform robotic surgery, such as the camera control and EndoWrist instrument manipulation and clutching. Currently, various robotic training platforms are available, but only the da Vinci Surgical Skills Simulator (dVSS; Intuitive Surgical) works with the original Si/Xi console, guaranteeing a more realistic experience. The simulation represents an important first step in training, to make actions at robotic console instinctive and to reduce, after an effective preparation, the operating time and the potential surgical errors (8).

To further support the surgeon formation, the double console with the da Vinci system has been available since 2009. During the robotic surgical procedure, the observers (students, residents, learning surgeons) can follow the operation from a second console, with the same visual perception of the surgeon. Furthermore, during robotic training, the dual console gives to the skilled surgeon the possibility to assist comfortably the "in-training" surgeon, being able to take the control of the robotic instruments. Thus, the trainee can gain a complete surgical practice, resulting in earlier proficiency. Some authors have reported the impact of the double console in surgical practice, showing that the dual-console encourages training and supervision by the proctor, with a high level of safety, good technical results and operating times $(9,10)$.

In parallel to the training of the robotic surgeon, the education of all robotic team members could be planned. In fact, an important role during the surgical procedure is carried out also by the assistant surgeon, which must actively participate and be able to face any problems, the nurses, which should be acquainted with the robotic system and the instruments, the anesthesiologists, which must manage the airway, the $\mathrm{CO}_{2}$ use and the unforeseen situations during the operation. Creating an expert group 


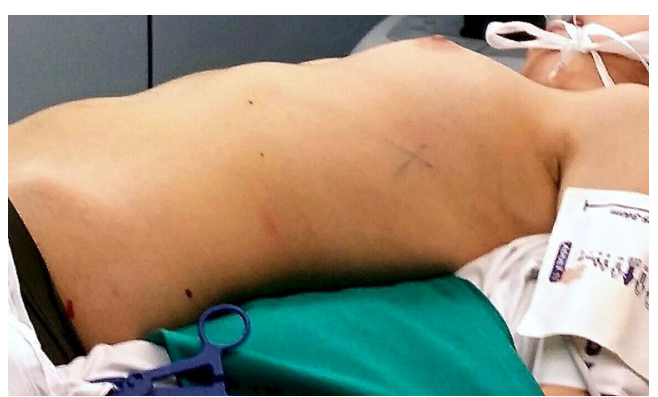

Figure 1 Position of patient during robotic thymectomy.

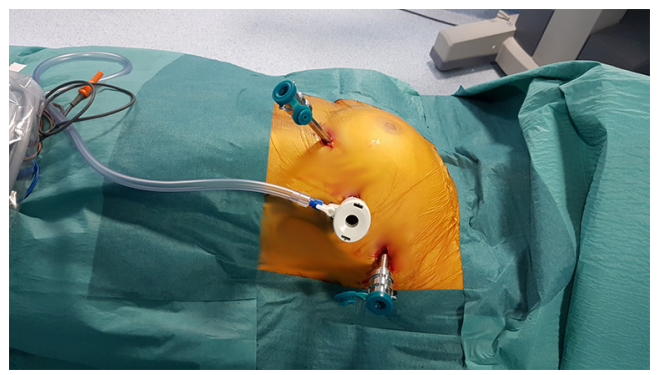

Figure 2 Robotic thymectomy port-mapping (left side access).

is the key to standardize the robotic technique, reducing the time and the cost of the procedures and increasing their efficacy and safety $(11,12)$.

The $\mathrm{CO}_{2}$ is useful to increase the working space into the chest cavity, but it can become an obstacle for contralateral lung ventilation and also be a cause of hemodynamic instability, due to a reduction of venous return and cardiac compliance.

\section{Robotic surgery in the anterior mediastinum}

From the earliest years of its application, focused on cardiac surgery, the robotic system appeared as fitting for the treatment of mediastinal lesions. The most frequent robotic mediastinal procedure reported in the literature is thymectomy, described for the first time by Yoshino in 2001 (13). Thymectomy is indicated in the presence of a thymoma or myasthenia gravis (MG) diagnosis. Thymoma is a rare thymic tumour, requiring particular attention during its removal to avoid the risk of neoplastic cells dissemination (no-touch technique), and it can be associated with MG. MG, a neuromuscular disorder caused by an anomalous production of autoantibody by the thymus or by ectopic tissue in mediastinal fat, requires an extended thymectomy in order to obtain the complete remission of the disease. For their pathologic characteristics, thymic neoplasm and MG need a high level of accuracy in dissection during thymectomy.

Thanks to the excellent three-dimensional magnified vision and the wide range of instrument manoeuvrability, which contribute to smooth manoeuvres in a restricted area such as the anterior mediastinum, the robotic approach in thymectomy is associated with positive operative and postoperative results. Using the robotic approach, the surgeon can achieve a radical procedure, essential for improving the oncological outcomes in thymoma patients and a positive MG remission rate trend. Robotic surgery is increasingly becoming an affirmed approach for the treatment of MG and clinical I-II stages of thymoma, throughout the world.

To increase the safety of the procedure during robotic thymectomy, given the lack of tactile feedback, the surgeon can use the Firefly, which after the injection of ICG permits the identification of vessels and nerves by near-infrared fluorescence. In thymic surgery, the Firefly application is both described for the detection of a mediastinal tumour and its relationship with the adjacent structures or for the safe identification of phrenic nerve in presence of abundant fat tissue in the mediastinum $(14,15)$.

\section{Surgical technique}

After a double lumen tube intubation, for selective singlelung ventilation, the patient is placed in supine position, with the left arm in a flexed and lower position, to fully expose the left side of the chest, to maximize working space and to avoid patient injury. It is advisable to place the patient in a 30-degree anti-decubitus position and in $10^{\circ}$ of reverse Trendelenburg, to increase the exposition of the mediastinum (Figure 1). The Port mapping of thymectomy provide for three incisions: the first is in the $5^{\text {th }}$ intercostal space at the anterior axillary line for the camera, the second in the $3^{\text {rd }}$ intercostal space at the anterior middle axillary line, the last is located in the $5^{\text {th }}$ intercostal space at the midclavicular line. All the incisions are just along the submammary line (Figure 2). The use of $\mathrm{CO}_{2}$ insufflation $(\mathrm{P}=$ $5-10 \mathrm{mmHg}$ ) is suitable to obtain a wider field of view and more space for manoeuvrability of the robotic instruments.

After the positioning of the trocars, the robotic system is docked. With the Si system, the surgical cart is positioned in the opposite side of the operating table, behind the patient's head, in an oblique direction (Figure 3). While using the $\mathrm{Xi}$ system, the surgical cart is placed from the opposite side 


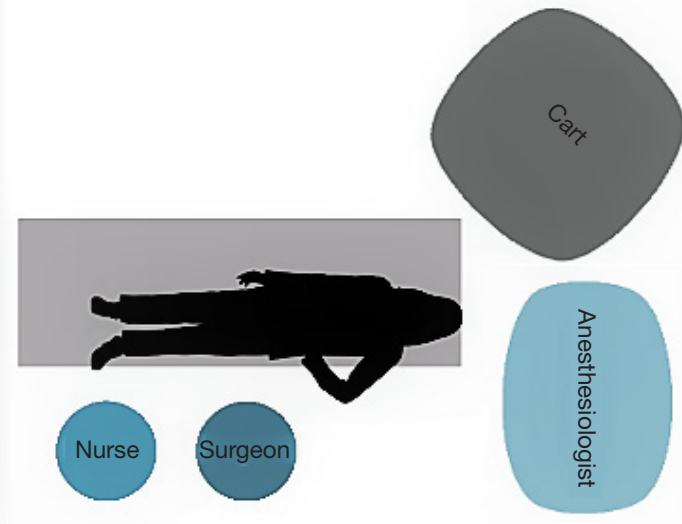

Figure $3 \mathrm{Si}$ system positioning for robotic thymectomy.

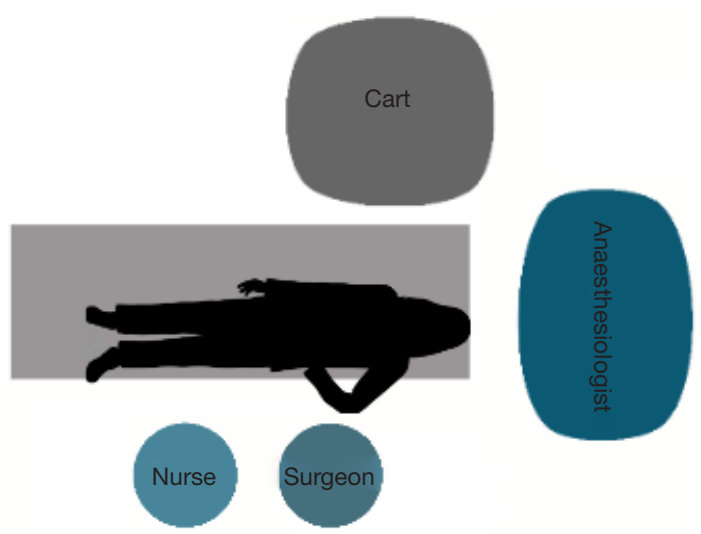

Figure $4 \mathrm{Xi}$ system positioning for robotic thymectomy.

of the patient and the correct placement is obtained thanks to auto-targeting with the camera pointed in the jugular area (Figure 4). With both systems the surgeon and scrub nurse work from the operative side of the patient, while the anesthesiologist stays near the head of the patient, to easily check his condition or the intubation.

During thymectomy, the instruments usually employed are monopolar (Spatula, EndoWrist; Intuitive Surgical, Inc., Sunnyvale, CA, USA) or bipolar (Maryland or Fenestrated Bipolar forceps, EndoWrist; Intuitive Surgical, Inc.). Although at the beginning it may seem more difficult to manage, the $30^{\circ}$ camera should be preferred, guaranteeing a better global vision of mediastinal structures.

Thymectomy can be performed by both sides, but most surgeons prefer the left-side approach. In this case, the dissection of the anterior mediastinal tissue starts from the bottom in retrosternal area, finding the contralateral mediastinal pleura and the right phrenic nerve, achieving a safe dissection of the right inferior horn, under direct vision of the nerve. Then, the surgeon proceeds to the left side, to the pericardial-phrenic angle and left phrenic nerve, upward until the identification of thymic superior horns. Then the thymic veins are carefully dissected, avoiding injury to the innominate vein, and separately clipped. The specimen is removed with an endoscopic bag from the cavity through one of the port incisions and a drainage tube is inserted in the chest cavity.

\section{Robotic surgery in lung cancer}

Despite the increasing use of the robotic system worldwide, the employment of robotic surgery in pulmonary resection for lung cancer has been characterized by a slower diffusion compared to mediastinal surgery, due to the fact that the open approach remains most commonly applied in nonsmall cell lung cancer (NSCLC) treatment.

Since 2001, when the first robotic lung lobectomy was reported, there has been considerable improvement of the robotic surgery application in the treatment of NSCLC. Several authors have conducted studies on the feasibility, safety, surgical and oncologic results of robotic lung resections (16).

The technologic evolution of the robotic system, associated with the increasing experience of the surgeons, have influenced the surgical results and consequently the indications.

In 2014, a comparison between patients underwent robotic lobectomy by da Vinci Standard system and S/Si system was published. The authors observed a reduction of mean operative time (237 vs. 172 minutes), of mean length of stay (4.4 vs. 3.8 days), of conversion ( $10.1 \%$ vs. $5.6 \%$ ), mortality $(1.4 \%$ vs. $0 \%)$ and morbidity rates $(22 \% v s$. $15 \%)$ using the latest system (17). The most recent studies confirmed satisfying post-operative results, in large series of patients. Nasir reported a median operative time of 107 minutes, a median hospital stay of 2 days, major morbidity in $9.6 \%$, a 30 -day operative mortality of $0.25 \%$ (18).

Various papers have reported reduced postoperative pain, shorter hospitalization, better aesthetic results in robotic surgery when compared to open surgery, with equivalent oncologic results. Kent analysed data of 33,095 patients underwent open or video-assisted thoracoscopic surgery (VATS) or robotic lobectomy and he observed a significant reductions in mortality, length of stay and overall complication rates in the robotic group, confirming the 
safety of robotic lobectomy (19).

The introduction of a new surgical technique in the oncologic field requires the analysis of oncologic results, to ensure the best prognosis to the patients. For this reason, some authors evaluated the nodal upstaging, which is considered a surrogate of oncologic quality of surgery, in robotic lobectomy, obtaining equivalent results to thoracotomy, in terms of dissected lymph nodes and nodal upstaging $(20,21)$.

Furthermore, in the last few years, data on oncologic outcomes of the application of robotic lobectomy in NSCLC patients have showed positive results, and confirm the possibility to obtain good oncologic radicality with robotic surgery. In a multicenter study from 2012, Park collected results of 325 early-stage NSCLC patients, underwent robotic lobectomy. The authors obtained a median operative time of 206 min, a median length of stay of 5 days and a morbidity rate of $25.2 \%$. From the oncologic point of view, five-year survival was $91 \%$ for stage IA, $88 \%$ for stage IB, $49 \%$ for stage II disease, while three-year survival for stage IIIA disease was $43 \%$ (22). In another multicenter study, after a median follow-up time of 30 months, the five-year stage-specific survival was $83 \%$ for stage IA, $77 \%$ stage IB, $68 \%$ for stage IIA, $70 \%$ for IIB, $62 \%$ for stage IIIA and $31 \%$ for stage IIIB (23). In our experience, with a median follow up time of 40.3 months, the overall survival at 60 months was $98.5 \%, 93.7 \%, 73.1 \%$ and $0 \%$ for stage I, II, III and IV respectively (24).

Given satisfying post-operative results and faster recovery, the thoracic surgeons are enlarging the indications of robotic surgery also to increasingly complicated patients, in opposite to the first part of the robotic lobectomy experience, when young patients without comorbidity were preferred. In 2017, Kass compared results of younger patients ( $<75$ years) with older patients ( $\geq 75$ years) underwent robotic lobectomy, concluding that robotic-assisted lobectomy is feasible and safe for patients of advanced age (25). In a recent study, the impact of obesity in robotic lobectomy was evaluated and no significant difference in intraoperative and postoperative results was showed, comparing obese patients with normal weight patients (26). In addition, the marginal pulmonary function was analyzed in relation to the approach, observing that robotic lobectomy potentially decreases the risk of postoperative lung complications, in particular in the patients with limited pulmonary function (27).

In view of these satisfying results, the indications for a robotic approach are being expanded, resulting today in the treatment of not only early stages of disease (28). Indeed, the improvements of the three-dimensional vision and the movements of instruments, associated with the recent introduction of the robotic staplers, enable to approach also complex cases, like locally advanced stages or following induction therapy, in a safe manner and with a rapid post-operative recovery. Therefore, thanks to the increased experience, diffuse pleural adhesions or chest wall involvement, vascular invasion, big pulmonary tumor, neo-adjuvant chemotherapy and/or radiation and previous thoracic surgery are no longer considered contraindications for robotic surgery (29-31).

\section{Surgical technique}

Over the years, the evolution of robotic technology in the different robotic systems has gone in parallel with the evolution of the surgical technique, reducing the invasiveness and therefore the traumatism for the patient. In fact, in the early years of its application in lung resections, the approach was almost similar to VATS, but with the upgrade of the robotic system, becoming progressively less bulky, the technique has also developed, providing to the surgeon the possibility to achieve a totally endoscopic operation. Different techniques and portmapping have been described by the various Authors, as a result of the evolution of the technique in parallel with the technology: Cerfolio reported a four-arm technique with an assistant port, Dylewski and Turner illustrated a threearm procedure with an assistant port and our technique consists in a four-arm totally endoscopic approach without an assistant port (32-34).

In the first part of our experience, about 20 years ago, when the robotic system was the da Vinci Standard, we developed a three-arm approach with an additional incision for the assistant surgeon. The patient was in lateral decubitus, with the operating table tilted at the tip of the scapula, like a posterolateral thoracotomy (Figure 5). The first port was placed at the $7^{\text {th }}-8^{\text {th }}$ space in the mid-axillary line (camera), the other at the $6^{\text {th }}-7^{\text {th }}$ intercostal space in the post-axillary line (left arm), a 'service entrance' was made at the $4^{\text {th }}-5^{\text {th }}$ intercostal space in the anterior axillary line (right arm), the assistant port was between the 'service entrance' and camera port (Figure 6) (16).

Then, with the introduction of da Vinci S, characterized by four operative arms, our technique was consequently modified and we applied a four-arm robotic approach with a utility port. The first $12 \mathrm{~mm}$ port was placed in the $7^{\text {th }}$ to 


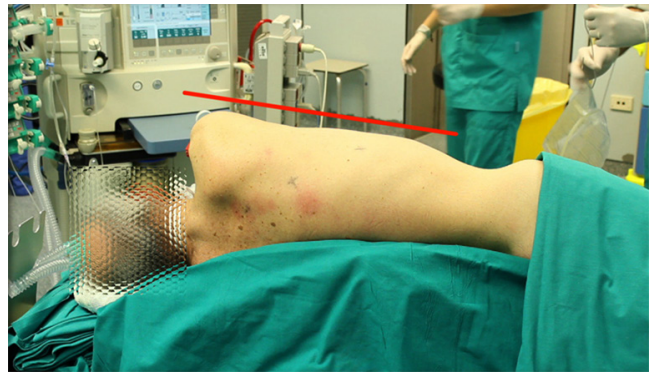

Figure 5 Position of patient during robotic lobectomy.

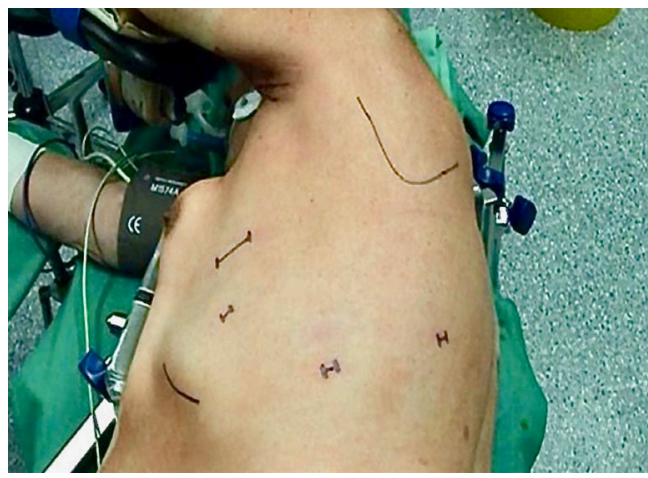

Figure 6 Robotic lobectomy port-mapping (three-arm approach).

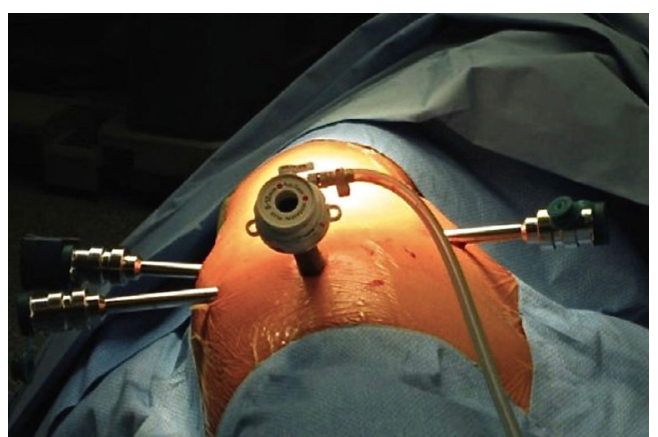

Figure 7 Robotic lobectomy port-mapping (four-arm approach).

$8^{\text {th }}$ intercostal space on the midaxillary line (camera), The second $8 \mathrm{~mm}$ port was in the $5^{\text {th }}-6^{\text {th }}$ intercostal space on the anterior axillary line, the third and the fourth $8 \mathrm{~mm}$ ports are respectively in the $6^{\text {th }}-7^{\text {th }}$ intercostal space on the posterior axillary line and in the auscultatory triangle. The utility port was positioned between the camera port and the anterior port. A distance of at least $6 \mathrm{~cm}$ between the ports was mandatory to avoid the collision of arms during the operation and $\mathrm{CO}_{2}$ was applied $(5-8 \mathrm{mmHg})$ to obtain the

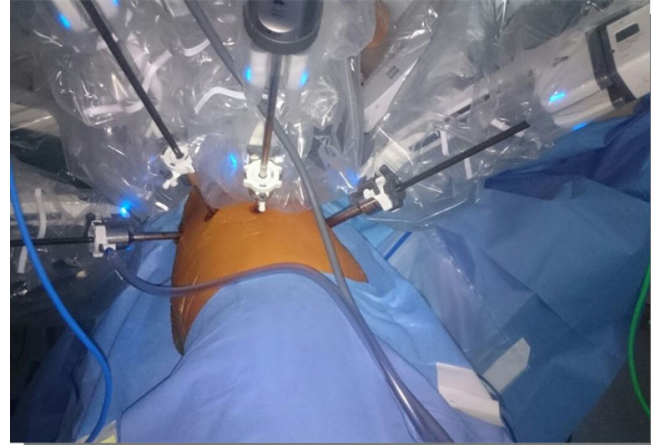

Figure 8 Robotic lobectomy port-mapping (totally endoscopic approach).

complete collapse of the lung, increasing the manoeuvring space (Figure 7) (35).

After the introduction of da Vinci Si, we standardized a four-arm "totally endoscopic" approach, without an assistant port. The camera port is positioned in the $7^{\text {th }}-8^{\text {th }}$ intercostal space on the posterior axillary line, the posterior ports are placed along the same intercostal space, the anterior port is in the lower position, in the intercostal space just over the diaphragm, on the anterior axillary line (Figure 8) (36).

Using da Vinci $\mathrm{Xi}$, it is possible to place the ports at closer distances and in the same intercostal space, reducing postoperative pain. Moreover, after the introduction of the robotic staplers, longer than the other robotic instruments, the surgical access incisions are moved as low as possible to increase the arm manoeuvrability.

Using Si system, the cart is positioned at head of the patient and the central point of its column must be in line with the longitudinal axis of the camera port. The correct distance between the cart and the patient is obtained when the marker on the camera arm is at the center of the blue line (Figure 9).

With the Xi system, the surgical cart can be placed either in back or in front of the patient.

When the robot is driven for docking, a laser line is activated to facilitate the correct placing and the laser crosshairs must correspond with the camera port. When the camera is inserted, looking towards the hilum, the autotargeting feature can be activated, achieving the optimal robotic arm placement (Figure 10).

The standardization of the instruments used during all major lung resections is recommended. The dissection of the hilar structures can be performed by use of monopolar (Hook or Scissors, Intuitive Surgical, Inc., Sunnyvale, CA, 


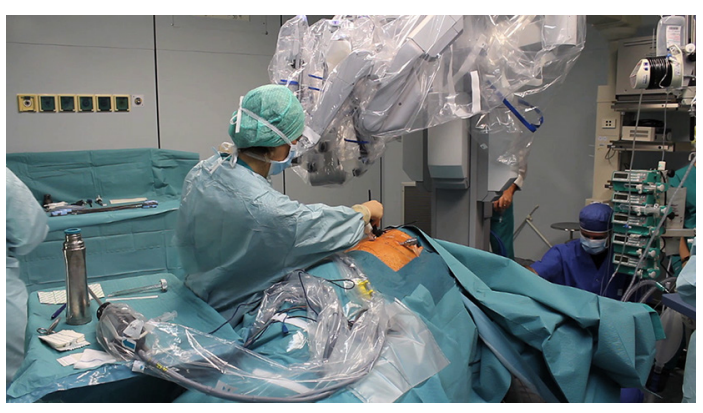

Figure 9 Si system positioning for robotic lobectomy.

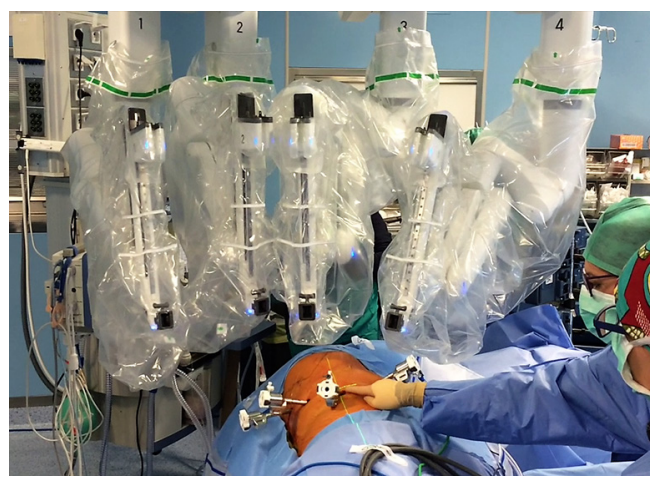

Figure $10 \mathrm{Xi}$ system positioning for robotic lobectomy.

USA) and/or bipolar instruments (Maryland, Intuitive Surgical, Inc., Sunnyvale, CA, USA), while a grasper (Cadiere, Prograsp, Intuitive Surgical, Inc., Sunnyvale, CA, USA) can be used to retract the lung.

Due to the technical characteristics of the robotic system, in particular the $3 \mathrm{D}$ vision and the large range of manoeuvrability of the robotic instruments, during the lobectomy the steps of dissection and section of hilar structures can be variable, avoiding the "obligatory direction", characteristic feature of the VATS.

\section{Conclusions}

Robotic surgery possesses the potential to become the standard of care for the surgical treatment of mediastinal lesions and lung cancer, in consideration of the achievable quality of surgery and radicality of resection. The technical features of the robotic system, among which the magnification of vision, tremor filter and an impressively wide range of instruments, are the most impressive and constitute a precious instrument to approach also advanced cancer in a safe way, with the advantages of minimally invasive technique.

As suggested by the latest NCCN guidelines, the minimally-invasive approach should be offered to all patients whenever possible (37). Therefore, thanks to the advanced robotic technology and to the standardization of surgical procedure, the robotic surgery may be able to offer to the patients a minimally invasive approach, respecting the surgical and oncologic principles.

\section{Acknowledgements}

None.

\section{Footnote}

Conflicts of Interest: Prof. F Melfi is an official proctor for Intuitive Surgical. The other authors have no conflicts of interest to declare.

\section{References}

1. Marino MV, Shabat G, Gulotta G, et al. From Illusion to Reality: A Brief History of Robotic Surgery. Surg Innov 2018;25:291-6.

2. Kalan S, Chauhan S, Coelho RF, et al. History of robotic surgery. J Robot Surg 2010;4:141-7.

3. Lane T. A short history of robotic surgery. Ann R Coll Surg Engl 2018;100:5-7.

4. Valero R, Ko YH, Chauhan S, et al. Robotic surgery: history and teaching impact. Actas Urol Esp 2011;35:540-5.

5. Ashrafian H, Clancy O, Grover V, et al. The evolution of robotic surgery: surgical and anaesthetic aspects. Br J Anaesth 2017;119:172-84.

6. Okusanya OT, Hess NR, Luketich JD, et al. Infrared intraoperative fluorescence imaging using indocyanine green in thoracic surgery. Eur J Cardiothorac Surg 2018;53:512-8.

7. Pearlstein DP. Robotic Lobectomy Utilizing the Robotic Stapler. Ann Thorac Surg 2016;102:e591-3.

8. Walliczek U, Förtsch A, Dworschak P, et al. Effect of training frequency on the learning curve on the da Vinci Skills Simulator. Head Neck 2016;38 Suppl 1:E1762-9.

9. Smith AL, Scott EM, Krivak TC, et al. Dual-console robotic surgery: a new teaching paradigm. J Robot Surg 2013;7:113-8.

10. Mikhail E, Salemi JL, Hart S, et al. Comparing Single and Dual Console Systems in the Robotic Surgical Training of Graduating OB/GYN Residents in the United States. 
Minim Invasive Surg 2016;2016:5190152.

11. Connor MA, Reinbolt JA, Handley PJ. Perioperative nurse training in cardiothoracic surgical robotics. AORN J 2001;74:851-7.

12. Carlos G, Saulan M. Robotic Emergencies: Are You Prepared for a Disaster? AORN J 2018;108:493-501.

13. Yoshino I, Hashizume M, Shimada M, et al. Thoracoscopic thymomectomy with the da Vinci computer-enhanced surgical system. J Thorac Cardiovasc Surg 2001;122:783-5.

14. Zirafa CC, Ricciardi S, Cavaliere I, et al. The application of robotic surgery on the anterior mediastinal tumors. J Vis Surg 2018;4:190.

15. Deen S, Farivar AS, Louie BE. Thoracic techniques: robotic thymectomy for thymoma. Indian J Surg Oncol 2013;4:132-7.

16. Melfi FM, Menconi GF, Mariani AM, et al. Early experience with robotic technology for thoracoscopic surgery. Eur J Cardiothorac Surg 2002;21:864-8.

17. Melfi FM, Fanucchi O, Davini F, et al. Robotic lobectomy for lung cancer: evolution in technique and technology. Eur J Cardiothorac Surg 2014;46:626-30; discussion 630-1.

18. Nasir BS, Bryant AS, Minnich DJ, et al. Performing robotic lobectomy and segmentectomy: cost, profitability, and outcomes. Ann Thorac Surg 2014;98:203-8; discussion 208-9.

19. Kent M, Wang T, Whyte R, et al. Open, video-assisted thoracic surgery, and robotic lobectomy: review of a national database. Ann Thorac Surg 2014;97:236-42; discussion 242-4.

20. Toosi K, Velez-Cubian FO, Glover J, et al. Upstaging and survival after robotic-assisted thoracoscopic lobectomy for non-small cell lung cancer. Surgery 2016;160:1211-8.

21. Zirafa C, Aprile V, Ricciardi S, et al. Nodal upstaging evaluation in NSCLC patients treated by robotic lobectomy. Surg Endosc 2019;33:153-8.

22. Park BJ, Melfi F, Mussi A, et al. Robotic lobectomy for non-small cell lung cancer (NSCLC): long-term oncologic results. J Thorac Cardiovasc Surg 2012;143:383-9.

23. Cerfolio RJ, Ghanim AF, Dylewski M, et al. The longterm survival of robotic lobectomy for non-small cell lung cancer: A multi-institutional study. J Thorac Cardiovasc Surg 2018;155:778-86.

24. Zirafa CC, Cavaliere I, Ricciardi S, et al. Long-term oncologic results for robotic major lung resection in nonsmall cell lung cancer (NSCLC) patients. Surg Oncol
2019;28:223-7.

25. Kass KS, Velez-Cubian FO, Zhang WW, et al. Effect of advanced age on peri-operative outcomes after roboticassisted pulmonary lobectomy: Retrospective analysis of 287 consecutive cases. J Geriatr Oncol 2017;8:102-7.

26. Montané B, Toosi K, Velez-Cubian FO, et al. Effect of Obesity on Perioperative Outcomes After Robotic-Assisted Pulmonary Lobectomy. Surg Innov 2017;24:122-32.

27. Kneuertz PJ, D'Souza DM, Moffatt-Bruce SD, et al. Robotic lobectomy has the greatest benefit in patients with marginal pulmonary function. J Cardiothorac Surg 2018;13:56.

28. Casiraghi M, Spaggiari L. Robotic resection of stage III lung cancer: an international retrospective study. J Thorac Dis 2018;10:S3081-3.

29. Lin MW, Kuo SW, Yang SM, et al. Robotic-assisted thoracoscopic sleeve lobectomy for locally advanced lung cancer. J Thorac Dis 2016;8:1747-52.

30. Chen S, Geraci TC, Cerfolio RJ. Techniques for lung surgery: a review of robotic lobectomy. Expert Rev Respir Med 2018;12:315-22.

31. Mariolo AV, Casiraghi M, Galetta D, et al. Robotic Hybrid Approach for an Anterior Pancoast Tumor in a Severely Obese Patient. Ann Thorac Surg 2018;106:e115-6.

32. Cerfolio RJ. Total port approach for robotic lobectomy. Thorac Surg Clin 2014;24:151-6, v.

33. Dylewski MR, Palacio J. Robotic Right Lower Lobectomy. Oper Tech Thorac Cardiovasc Surg 2017;22:17-42.

34. Turner SR, Latif MJ, Park BJ. Robotic assisted VATS lobectomy for loco-regionally advanced non-small cell lung cancer. Video-assist Thorac Surg 2017;2:10.

35. Melfi FM, Fanucchi O, Davini F, et al. VATS-based approach for robotic lobectomy. Thorac Surg Clin 2014;24:143-9, v.

36. Zirafa CC, Davini F, Romano G, et al. Robotic Lobectomy: Left Lower Lobectomy by Surgery. Oper Tech Thorac Cardiovasc Surg 2017;22:43-57.

37. NCCN Clinical Practice Guidelines in Oncology. Nonsmall cell lung cancer Guidelines. Version 3, 2019. January $18,2019$.

Cite this article as: Zirafa CC, Romano G, Key TH, Davini F, Melfi F. The evolution of robotic thoracic surgery. Ann Cardiothorac Surg 2019;8(2):210-217. doi: 10.21037/ acs.2019.03.03 